\section{CORRECTION FOR RESPIRATION ARTIFACT IN PULMONARY BLOOD PRESSURE SIGNALS OF VENTILATED PATIENTS}

\author{
Sebastiaan A. A. P. Hoeksel, MSc, ${ }^{1}$ \\ Johannes A. Blom, PhD, ${ }^{2}$ Jos R. C. Jansen, $P h D,{ }^{3}$ and \\ JanJ. Schreuder, $M D, P h D^{1}$
}

Hoeksel SAAP, Blom JA, Jansen JRC, Schreuder JJ. Correction for respiration artifact in pulmonary blood pressure signals of ventilated patients.

J Clin Monit 1996; 12: 397-403

ABSTRACT. Objective. To develop an algorithm that corrects pulmonary artery pressure signals of ventilated patients for the respiration artifact. The algorithm should test the validity of the pulmonary pressure signal and differentiate between the cyclic respiration artifact and true measurement artifacts. Methods. The shape of each pulmonary pressure beat is described by eight characteristic features, including mean pressure value and the systolic and diastolic timing and pressure values. The features are corrected for the respiration artifact by fitting them in a least-squares sense on the first and second harmonics of the ventilator frequency. The corrected features are used by a signal validation algorithm, which adds a validity flag to each pressure beat. The validation algorithm rejects pressure beats with sudden changes in their shape but adapts itself when the changes persist. Results. The performance of the correction and validation technique was evaluated using pulmonary artery pressure signals of 30 patients who were scheduled for open heart surgery. The algorithm correctly recognized as invalid data those pressure signals disturbed by coagulation, surgical manipulations, or flushes of the pressure line. The algorithm marked on average $77 \pm 11 \%$ of the pulmonary pressure beats as valid. Conclusions. The validation algorithm marked sufficient pressure beats as valid to update a trend display every $5 \mathrm{sec}$. The correction algorithm enabled the validation algorithm to differentiate between true measurement artifacts and the respiration artifact.

KEY WORDS. Algorithm: signal processing, data recording, mechanical ventilation, pulmonary pressure.

\section{INTRODUCTION}

The reliability of information provided by monitoring equipment is vital for the treatment of the critical care patient. The aspect of signal reliability has gained even more importance with the introduction of closed-loop systems in a clinical environment [1,2]. Artifact rejection. algorithms ensure that closed-loop systems do not respond to any non-physiological change in the measured input signal.

Arterial blood pressure is the most frequently used

From the ${ }^{1}$ Department of Anaesthesiology, Cardiovascular Research Institute Maastricht, University of Limburg, Maastricht, The Netherlands, ${ }^{2}$ Department of Electrical Engineering, Eindhoven University of Technology, The Netherlands, and the ${ }^{3}$ Department of Pulmonary Diseases, Erasmus University, The Netherlands.

Received Jan 16, 1996, and in revised form May 3, 1996. Accepted for publication Jun 13, 1996.

Address correspondence to Sebastiaan A.A.P. Hoeksel, MSc, Department of Anacsthesiology, Cardiovascular Research Institute Maastricht, University of Limburg, PB 5800, 6202 AZ, Maastricht, The Netherlands. feedback signal [3-5] in a clinical setting. Blood pressure signals can be distorted by air bubbles or blood clotting in the pressure line, electromagnetic interference with the coagulation equipment, surgical interventions, movement of the patient, blood sampling, and flushing of the pressure line. Under physiological circumstances the shape of an arterial pressure beat does not change drastically, thus detection of artifacts is possible using pattern recognition to detect and reject those pressure beats that differ sufficiently from previous beats. 


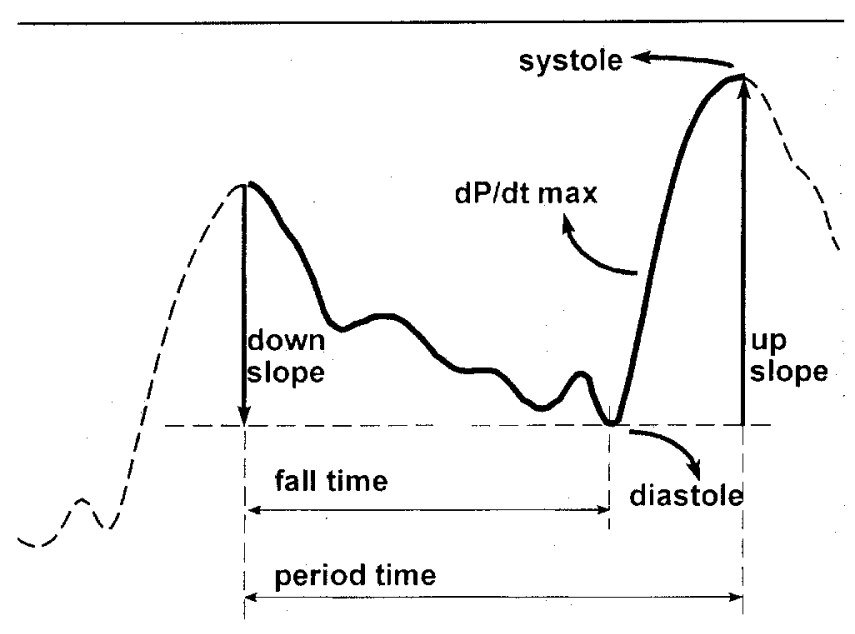

Fig 1. The shape of a pulmonary pressure beat is described by eight characteristic features. MPAP is the average pressure between two successive systoles.

The validation of pulmonary artery pressure signals is more complicated than the validation of systemic arterial pressure signals because the former are changed by the respiration artifact. We developed a filtering technique that corrects characteristic features of pulmonary pressure beats for the respiration artifact during mechanical ventilation. The corrected features are used to test the validity of each pulmonary pressure beat on a beat-tobeat basis. As a possible application of the algorithm we designed a $5 \mathrm{sec}$ trend display of the validated average pulmonary pressure.

\section{ALGORITHM}

The algorithm can be separated into three parts: the first part of the algorithm detects each beat in the pulmonary artery pressure signal and extracts characteristics features; the second part corrects these characteristic features for the respiration artifact; and the last part validates each pulmonary pressure beat using the corrected features.

\section{Extraction of features from the pulmonary pressure signal}

The first part of the algorithm detects pressure beats in the pulmonary pressure signal. The digitized pressure signal is passed through a lowpass third-order Butterworth filter with its cut-off frequency at $10 \mathrm{Hertz}$. This filter provides a smooth pressure signal and does not significantly suppress systolic pressure values. Local minima and maxima in the pressure signal are detected when its first-order time-derivative crosses zero. At each maximum an upstroke pressure is calculated as the difference between the current maximum and the last minimum. A new systole is detected if the upstroke pressure is larger than $80 \%$ of the moving average of previous systolic upstroke pressures.

We defined eight characteristic features to describe the shape of a pulmonary pressure beat (Figure 1). A beat is defined between two systoles since each new beat is detected at its systole. The more commonly used time period between two consecutive diastoles can, however, be derived from the timing features of two successive periods. Mean pulmonary artery pressure (MPAP) is the average pressure value between two consecutive systoles. Systolic and diastolic pressures follow from the maximum and minimum of the systolic upstroke. Up slope and down slope pressures are derived from the diastolic pressure of the current period and the systolic pressures of the previous and the current periods. The period time is the time between two consecutive systoles and the fall time is the time difference between the start of the period and the diastole of that period. $d P / d t_{\max }$ is found by continuously updating it during the systolic upstroke with each $d P / d t$ larger than the current $d P / d t_{\max }$.

\section{Filtering of pressure beats}

The features derived from pulmonary pressure beats show a strong fluctuation with the ventilator signal. Mechanical ventilation causes fluctuations in intra-thoracic pressures, which influence the venous return, the filling of the atria, and the impedance of the pulmonary vascular bed [6]. As a consequence, both offset and pulse pressure of the pulmonary artery pressure signal fluctuate during ventilation. The features should be corrected for this fluctuation to enable distinction between the respiration artifact and true measurement artifacts. Averaging of the features will not work since this will also filter out measurement artifacts. The periodic property of the respiration artifact in ventilated patients can be used to describe a feature $y$ by a DC term plus a sine with $m$ higher harmonics and an unknown phase shift,

$\gamma\left(t_{n}\right)=\frac{a_{0}}{2}+\sum_{k=1}^{m}\left(a_{k} \cos \left(\omega k t_{n}\right)+b_{k} \sin \left(\omega k t_{n}\right)\right)+e\left(t_{n}\right)$

where $\omega=2 \pi f$ and $e\left(t_{n}\right)$ is a random noise process. The ventilator frequency $f$ can either be read from the ventilator or extracted from the fluctuations in the pulmonary pressure signal. The variables $a_{0}, a_{k}$, and $b_{k}$ are allowed to vary slowly in time to reflect changes in the patient's condition.

Rather than estimating all parameters simultaneously, $a_{0} / 2$ is determined using a lowpass filter and then $a_{k}$ and 
$b_{k}$ are determined using a least-squares fitting procedure. The lowpass filter is implemented as the integral over one ventilator period divided by the period time of one ventilator cycle,

$\frac{a_{o}}{2}=\frac{1}{T} \int_{t_{n}-T}^{t_{n}} y(\tau) d \tau$

where $T=1 / f$. Integral (2) is solved numerically using linear interpolations. The ventilator frequency is calculated counting the number of positive zero-crossings of the lowpass filtered MPAP feature with the MPAP feature itself [7].

Preliminary results indicated that for the estimation of $a_{k}$ and $b_{k}$ in (1) only the first 2 harmonics (i.e. $m=2$ ) would suffice. Given $a_{0} / 2$ from (2), the parameters $a_{k}$ and $b_{k}$ can be found by minimizing the cost function

$J\left(\theta, t_{n}\right)=\sum_{k=1}^{n} \lambda^{n-k}\left[\gamma\left(t_{k}\right)-\frac{a_{0}}{2}-\theta\left(t_{k}\right)^{T} \phi\left(t_{k}\right)\right]^{2}$

where

$\phi\left(t_{n}\right)=\left[\sin \left(\omega t_{n}\right) \cos \left(\omega t_{n}\right) \sin \left(2 \omega t_{n}\right) \cos \left(2 \omega t_{n}\right)\right]^{T}$

$\theta\left(t_{n}\right)=\left[a_{1}\left(t_{n}\right) b_{1}\left(t_{n}\right) a_{2}\left(t_{n}\right) b_{2}\left(t_{n}\right)\right]^{T}$

The weighing factor $\lambda^{\mathrm{n}-\mathrm{k}}$ depends on a forgetting factor $\lambda$ and is added to allow the algorithm to track changes in the true parameter vector $\theta_{t}$. In this way the effect of previous measurements on the cost function $J\left(\theta, t_{n}\right)$ decays exponentially. The recursive estimation of the parameter vector $\theta$ that minimizes the cost function $J\left(\theta, t_{n}\right)$ is given by Ljung [8] as

$\hat{\theta}\left(t_{n}\right)=\hat{\theta}\left(t_{n-1}\right)+L\left(t_{n}\right)\left[y\left(t_{n}\right)-\phi^{T}\left(t_{n}\right) \hat{\theta}\left(t_{n-1}\right)\right]$

$L\left(t_{n}\right)=P\left(t_{n}\right) \phi\left(t_{n}\right)$

$P\left(t_{n}\right)=\frac{1}{\lambda}\left[P\left(t_{n-1}\right)-\frac{P\left(t_{n-1}\right) \phi\left(t_{n}\right) \phi\left(t_{n}\right)^{T} P\left(t_{n-1}\right)}{\lambda+\phi^{T}\left(t_{n}\right) P\left(t_{n-1}\right) \phi\left(t_{n}\right)}\right]$

The convergence of the parameter vector $\hat{\theta}$ towards the true parameter vector $\theta_{t}$ will depend on the statistical properties of the noise process $e\left(t_{n}\right)$ [8]. The estimation of $\theta_{t}$ using (6), (7) and (8) can be reduced to 4 separate estimations of $a_{1}, b_{1}, a_{2}$ and $b_{2}$, since sine and cosine are orthogonal functions, thus eliminating the computational efforts of manipulating $4 \times 4$ matrices.

The forgetting factor $\lambda$ of the least-square fitting algorithm determines the weight that is given to passed measurements in the cost function (3). A value of $\lambda$ that is too high will give a high weight to passed measure- ments making the convergence of $\hat{\theta}$ towards $\theta_{\mathrm{t}}$ slow, whereas a value of $\lambda$ that is too low will make the estimations noise-sensitive. The final choice of $\lambda$ is given by this balance between tractability and noise-sensitivity. Model simulations using a known set of parameters $a_{k}$ and $b_{k}$ corrupted by white noise were used to obtain an optimal choice of $\lambda$. Based on these simulation studies a value of 0.95 was chosen for $\lambda$.

The estimated parameters $a_{k}$ and $b_{k}$ can be used to calculate the pulmonary features corrected for the modulation by the ventilator as

$y\left(t_{n}\right)-\sum_{k=1}^{2}\left(a_{k} \cos \left(\omega k t_{n}\right)+b_{k} \sin \left(\omega k t_{n}\right)\right)$

The features $y_{M P A P}, y_{s y s}, y_{\text {dias }}$ and $\gamma_{d P / d t}$ are corrected using (9). The corrected features of $y_{u p}$ and $y_{\text {down }}$ are calculated from the corrected features of $y_{s y s}$ and $y_{\text {dias }}$. The timing features of a beat $\gamma_{\text {period }}$ and $y_{\text {fall }}$ are minimally modulated by the ventilator signal and do not require correction for the respiration artifact.

\section{Determination of end-expiratory feature values}

In the ventilated patient, one is also interested in the endexpiratory pressure values since here the condition of stable airway pressure is most likely to be met. Standard monitoring equipment approximates these end-expiratory values using a weighted filtering technique, which favours end-expiratory values over non end-expiratory values [9]. End-expiratory values are identified by analyzing the beat-to-beat difference, assuming that the MPAP is relatively stable at end-expiration and relatively unstable elsewhere $[9,10]$. Using the previously described estimation technique, however, the function describing the respiration artifact is known at all times and can be used to calculated the end-expiratory values with each new pulmonary pressure beat.

\section{Validation of pulmonary pressure}

The corrected features of a beat are used to add a validity flag to that beat. All pressure beats with their corrected features within an acceptable range are used to update a moving average model of each feature using

$y_{\text {model }}\left(t_{n}\right)=\gamma_{\text {model }}\left(t_{n-1}\right) * 0.9+\gamma\left(t_{n}\right) * 0.1$

The acceptable range is defined as the absolute mininum and maximum values of each feature (Table 1). The factor 0.9 is chosen so that changes are tracked in time without 
Table 1. The initially used validation boundaries, the acceptable range of each feature, and the validation boundaries as determined after evaluating the results of all 30 patients. The validation boundaries are the maximally allowed deviations of each feature from the corresponding feature of the beat model

\begin{tabular}{llll}
\hline & $\begin{array}{l}\text { Initial } \\
\text { validation } \\
\text { boundaries }\end{array}$ & $\begin{array}{l}\text { Acceptable } \\
\text { range }\end{array}$ & $\begin{array}{l}\text { Validation } \\
\text { boundaries }\end{array}$ \\
\hline MPAP, mmHg & 2 & $2-60$ & 1.10 \\
Sys, mmHg & 2 & $5-70$ & 1.85 \\
Dias, mmHg & 2 & $1-50$ & 1.50 \\
Up pulse, mmHg & 3 & $3-30$ & 1.95 \\
Down pulse, mmHg & 3 & $3-30$ & 2.13 \\
dP/dt max $_{\text {m mHg sec }}{ }^{-1}$ & 100 & $25-500$ & 23.8 \\
Period time, msec & 120 & $20-200$ & 140 \\
Fall time, msec & 120 & $15-180$ & 140 \\
\hline
\end{tabular}

tracking the ventilatory disturbances. The beat model now stores the history of the previous feature values. A beat is considered valid if its feature values are within the acceptable range and if they do not deviate too strongly from the previous feature values. In this way, a pressure beat with a sudden change in its shape will be rejected because it deviates from the beat model, whereas the beat model will slowly converge towards a new state if the change in shape is permanent. The maximally allowed deviation of the feature values from the beat model will be referred to as the validation boundaries. Initial estimates of the validation boundaries were used because no prior knowledge of the beat-to-beat difference was available (Table 1). Both the acceptable range and the initial estimates of the validation bounderaries were chosen after interviewing an experienced clinician and analyzing results of a pilot study.

\section{EVALUATION OF PERFORMANCE}

The performance of the proposed filtering and validation technique was evaluated using data from 30 patients scheduled for cardiac surgery. Informed patient consent was obtained the evening before surgery. The pulmonary artery pressure signal was obtained using a pressure transducer (Uniflow ${ }^{\text {(i) }}$, Baxter) connected to a 7 F Swan-Ganz pulmonary catheter, which was introduced prior to surgery via the right jugular vein. The analog pressure signal was taken from the operating room monitoring system's (M1168A Component Monitoring System, HP) pressure modules (M1006A, HP), which had a built-in lowpass filter at a frequency of 12.5 Hertz. The algorithms were implemented on an IBM-AT-compatible personal computer. The analog pressure signal was im-
Table 2. Results of the correction algorithm in 30 patients. Shown are the estimated amplitudes of the first and second harmonics and the corrections necessary to calculate the end-expiratory values from the corrected features. All values are mean $\pm S D$

\begin{tabular}{lll}
$\begin{array}{l}\text { Amplitude } \\
\text { first }\end{array}$ & $\begin{array}{l}\text { Amplitude } \\
\text { second } \\
\text { harmonic }\end{array}$ & $\begin{array}{l}\text { Corrections re- } \\
\text { quired to obtain } \\
\text { end-expiratory } \\
\text { values }\end{array}$ \\
\hline
\end{tabular}

\begin{tabular}{|c|c|c|c|}
\hline$M P A P, \mathrm{mmHg}$ & $1.89 \pm 0.54$ & $0.55 \pm 0.19$ & $1.66 \pm 0.45$ \\
\hline Sys, $\mathrm{mmHg}$ & $1.86 \pm 0.63$ & $0.72 \pm 0.21$ & $1.81 \pm 0.54$ \\
\hline $\begin{array}{l}\text { Dias, mmHg } \\
\mathrm{dP} / \mathrm{dt} \text {. }\end{array}$ & $2.21 \pm 0.65$ & $0.72 \pm 0.22$ & $1.92 \pm 0.51$ \\
\hline $\mathrm{mmHg} \mathrm{sec}-1$ & $10.9 \pm 4.1$ & $5.8 \pm 2.4$ & $1.9 \pm 0.5$ \\
\hline
\end{tabular}

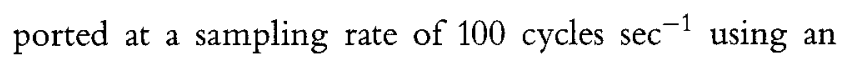
analog-to-digital convertor (DAS1600, Keithley Metrabyte) with an accuracy of 12 bits for $500 \mathrm{mmHg}$. Programming was done in Turbo Pascal v.7.0 (Borland Pascal, Scotts Valley, CA). We designed a trend display that was updated every $5 \mathrm{sec}$ with the averaged MPAP feature. A 5-sec epoch was considered valid if at least 2 periods were found to be valid.

During the surgical procedure the pulmonary artery pressure waveform and the $5 \mathrm{sec}$ trend display were observed by an experienced clinician who made notes of all relevant events. The validation algorithm correctly recognized pressure signals disturbed by coagulation, surgical manipulations, or flushes of the pressure line as invalid data and did not mark any 5 sec interval incorrectly as valid data. However, the system did mark successive $5 \mathrm{sec}$ episodes as invalid when the signal was judged valid by the clinician. This occurred only during atrial fibrillation; here the validation boundaries of the timing features were chosen too small. A representative example of the performance of the filtering algorithm during a stepwise change in ventilatory frequency is shown in Figure 2.

All feature values of each beat were stored on hard-disk for off-line analysis. For all patients the average amplitudes of the first and second harmonic of each feature were calculated as $\sqrt{a_{1}^{2}+b_{1}^{2}}$ and $\sqrt{a_{2}^{2}+b_{2}^{2}}$ (Table 2). Also shown in Table 2 are the average values that were subtracted from each corrected feature to obtain its endexpiratory value. The average deviations of the feature values from the beat model have been calculated for all 30 patients and are given in Table 3.

The percentage of valid periods, when the validation boundaries were chosen at $n$ times the average deviations found in these 30 patients, was calculated for $n$ ranging from 0.5 to 5 (Figure 3). In general, the chance that invalid data is incorrectly identified as valid increases for values of $n$ that are too large, whereas for values of $n$ that 


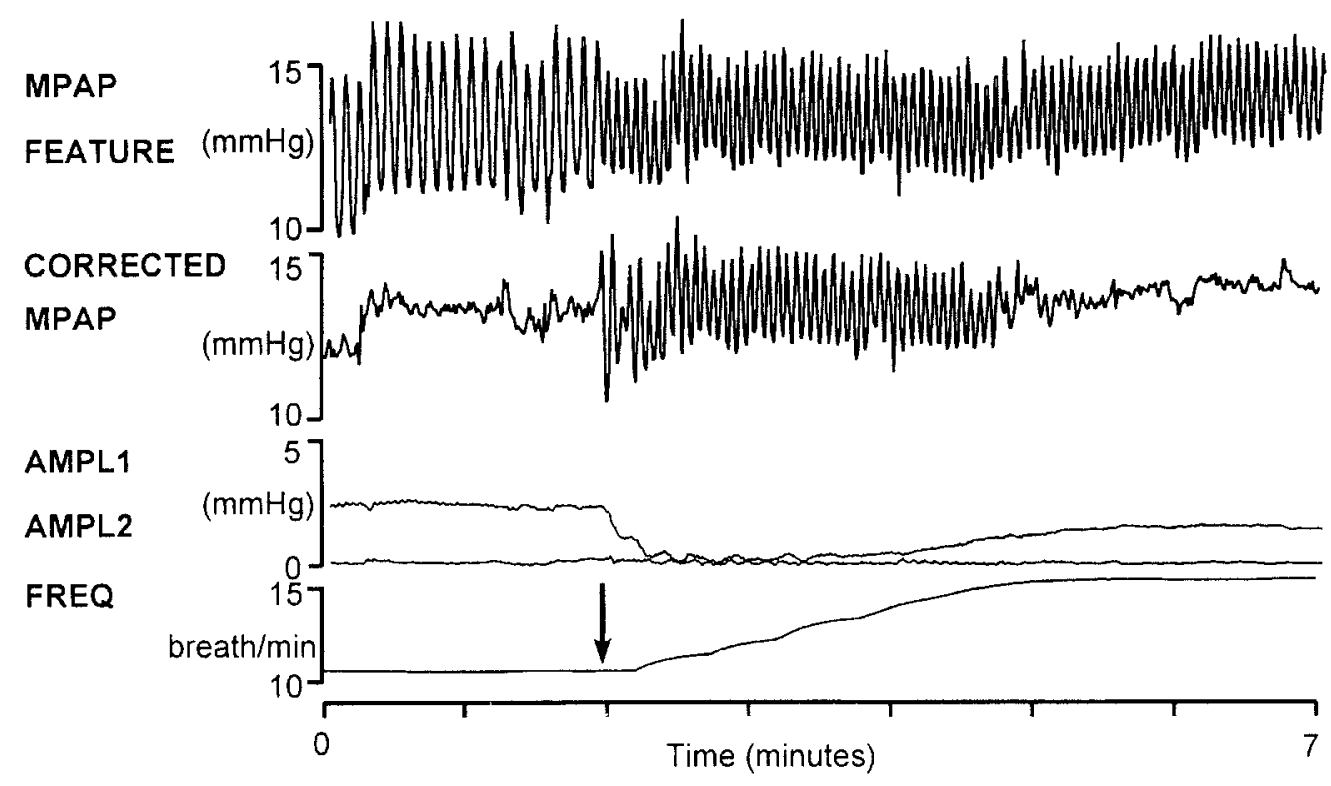

Fig 2. The performance of the correction algorithm for the MPAP feature during a step-wise change in ventilator frequency. The top panel shows the fluctuation of the MPAP feature with the ventilatory frequency. The middle panels show the MPAP feature corrected for the respiration artifact and the amplitudes of the first (AMPL1) and second (AMPL2) harmonics as estimated by the algorithm. The lower panel shows the estimated ventilator frequency (FREQ), which is increased step-wise from 12 to 15 breaths $\mathrm{min}^{-1}$ at the arrow. It takes almost 3 min before the new ventilator frequency has been identified. During this period of time correction is not possible.

are too low, insufficient periods will be marked as valid. With this dualism and the intended application (a closedloop system) in our minds, we have chosen the validation bounderies for $n$ as 2.5 . With these new validation boundaries (Table 1) $77 \pm 11 \%$ (mean \pm SD) of the periods were found to be valid, while $85 \pm 13 \%$ of the 5 sec periods were valid. We also investigated whether the percentage of valid periods is biased on ventilator cycle. The ventilatory phase was extracted from the first harmonic of the MPAP feature. The percentage of valid

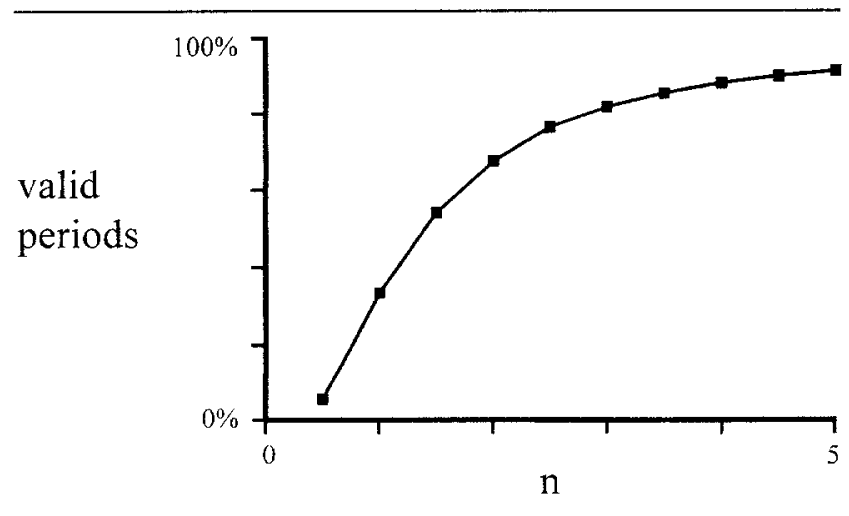

Fig 3. The percentage of valid periods when the validation boundaries are varied over $\mathrm{n}$ times (from 0.5 till 5 in steps of 0.5 ) the average deviations from the beat model as found in 30 patients. periods was calculated for eight different intervals in the ventilatory cycle (Figure 4).

\section{DISCUSSION}

The proposed algorithm provided a simple way to correct characteristic features of pulmonary pressure beats for the cyclic respiration artifact. We chose not to correct the pulmonary pressure waveform because this would require a time-consuming filtering technique. Pulmonary pressure beats were validated by comparing their corrected features with the averaged features of the previous pressure beats. The validation boundaries could be chosen sufficiently small because the noise caused by the respiration artifact had been reduced by the filtering algorithm (Table 3). The algorithm identified a sufficient percentage of pressure beats as valid to update a trend display every $5 \mathrm{sec}$, while correctly recognizing pressure signals disturbed by coagulation, surgical manipulations, or flushes of the pressure line as invalid data.

The proposed filtering algorithm can be seen as a sharp bandstop filter around the ventilator frequency and its higher harmonics. The sharpness of this bandstop filter is mainly determined by the forgetting factor $\lambda$ of the least-squares fitting algorithm, which allows the parameters $a_{k}$ and $b_{k}$ to create a time-varying phase and, 
Table 3. Reduction in the difference between the features of a beat and the corresponding features of the beat model, when the non-corrected and the corrected features of that beat are used. The average improvement is expressed as $\left(1-\Delta y_{c} / \Delta y\right) 100 \%$, where $\Delta y$ is the difference using the non-corrected and $\Delta y_{c}$ is the difference using the corrected feature

\begin{tabular}{|c|c|c|c|}
\hline & Features & $\begin{array}{l}\text { Corrected } \\
\text { features }\end{array}$ & $\begin{array}{l}\text { Average } \\
\text { improve- } \\
\text { ment }\end{array}$ \\
\hline$\triangle \mathrm{MPAP}, \mathrm{mmHg}$ & $1.39 \pm 0.33$ & $0.44 \pm 0.13$ & $68 \%$ \\
\hline$\Delta$ sys, mmHg & $1.56 \pm 0.43$ & $0.74 \pm 0.25$ & $53 \%$ \\
\hline$\Delta$ dias, $\mathrm{mmHg}$ & $1.68 \pm 0.41$ & $0.60 \pm 0.17$ & $64 \%$ \\
\hline$\Delta \mathrm{dP} / \mathrm{dt}, \mathrm{mmHg} \mathrm{sec}^{-1}$ & $13.2 \pm 4.9$ & $9.5 \pm 3.9$ & $28 \%$ \\
\hline$\Delta$ up, $\mathrm{mmHg}$ & $1.30 \pm 0.42$ & $0.78 \pm 0.31$ & $40 \%$ \\
\hline$\Delta$ down, $\mathrm{mmHg}$ & $1.36 \pm 0.41$ & $0.85 \pm 0.30$ & $38 \%$ \\
\hline$\Delta$ period, msec & $54.8 \pm 24.2$ & - & - \\
\hline$\Delta$ fall, msec & $56.5 \pm 22.1$ & - & - \\
\hline
\end{tabular}

ampl
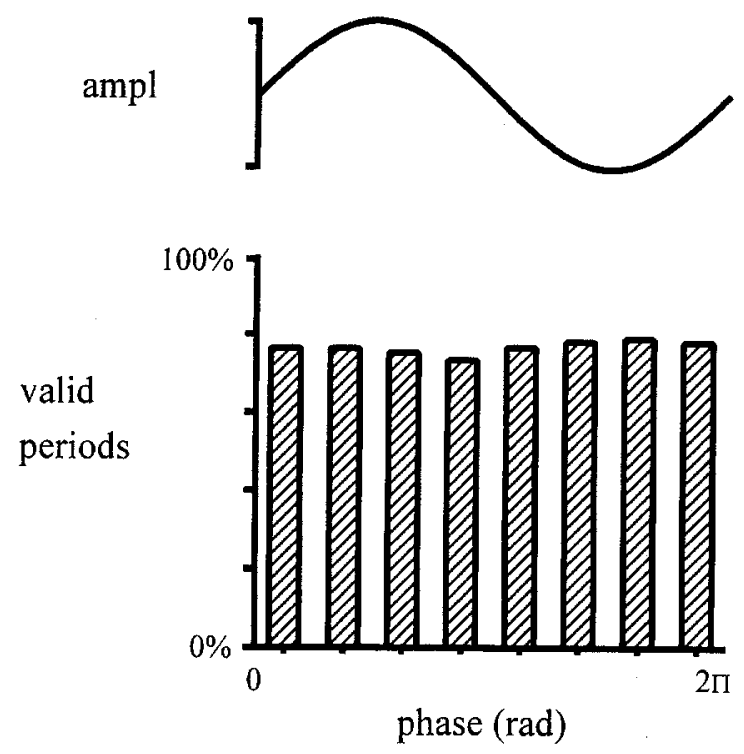

Fig 4. The percentage of valid periods as a function of the phase in the ventilator cycle. The current phase of the ventilator cycle was extracted using the first harmonic of the MPAP feature. The top panel schematically shows this harmonic, the lower panel shows the percentage of valid periods in each phase. The validation boundaries, which were determined after analyzing the results of all 30 patients (Table 1), were used.

thus, a frequency shift related to the first-order timederivative of this phase. The algorithm should not be mistaken for an ordinary bandstop filter, because a feature will only be corrected for the respiration artifact when it is consistently present in the pulmonary pressure signal; no correction will be made when the cyclic ventilator signal is not clearly present in the pulmonary pressure signal. In an alternative implementation, the time required for the algorithm to find the ventilator frequency in the pulmonary pressure signal (Figure 2), can be eliminated by reading it directly from the ventilator.

We validated pulmonary pressure beats by analyzing the fluctuations in eight characteristic features. Neural networks have been proposed to validate the shape of pressure signals $[11,12]$. Their disadvantages are that online learning is required and that their inference process is not as transparent. Online model identification [13] and spectral density estimation [14] have also been suggested for the analysis of the shape of pressure waveforms. Using these techniques, detection of invalid measurements will, however, be delayed because multiple pressure samples need to be evaluated before a change in the pressure waveform becomes apparent.

The proposed correction and validation algorithm may have several applications. It can be used to calculate end-expiratory values with each new pressure beat. The validity flag set by the algorithm can be used to filter out measurement artifacts in long-time trend recordings and can thus assist in the automated processing of pressure recordings. The corrected pulmonary features provide a smoother trend recording compared with algorithms that average pulmonary features over a fixed period of time. Finally, the validated pulmonary pressure can be used as a feedback signal in a closed-loop system where an artifact-free signal is crucial.

\section{REFERENGES}

1. O'Hara DA, Bogen DK, Noordergraaf A. The use of computers for controlling the delivery of anesthesia. Anesthesiology 1992; 77: 563-581

2. Linkens DA, Hacisalihzade SS. Computer control systems and pharmacological drug administration: A survey. J Med Eng Tech 1990; 14: 41-54

3. Colvin JR, Kenny GNC. Automatic control of arterial pressure after cardiac surgery, evaluation of a microcomputer-based control system using glyceryl trinitrate and sodium nitroprusside. Anaesthesia 1989; 44: 37-41

4. Martin JF, Smith T, Quinn ML, Schneider AM. Supervisory adaptive control of arterial pressure during cardiac surgery. IEEE Trans Biomed Eng 1992; 39: 389-393

5. Chitwood WR, Cosgrove DM III, Lust RM, the Titrator Multicenter Study Group. Multicenter trial of automated nitroprusside infusion for postoperative hypertension. Ann Thorac Surg 1992; 54: 517-522

6. Foëx P. The mechanical effects of raised airway pressure. In: Prys-Roberts C, ed. The circulation in anaesthesia. Oxford: Blackwell Scientific Publications, 1980: 281-294

7. Papoulis A. Probability, random variables, and stochastic processes. Singapore: McGraw-Hill, 1991: 603-612 
8. Ljung L, Söderström T. Theory and practice of recursive identification. The Massachusetts Institute of Technology: MIT Press, 1983

9. Hewlett Packard. An algorithm for the reduction of the respiration artifact in pulmonary artery pressure measurements, 1989, Application note: 5953-7352

10. Ellis DM. Interpretation of beat-to-beat blood pressure values in the presence of ventilatory changes. J Clin Monit 1985; $1: 65-70$

11. Odaka $T$, Agata $H$, Furutani $H$, Ogura H. A general purpose neural network simulator system for medical data processing. J Med Sys 1994; 18: 305-314

12. Pike T, Mustard A. Automated recognition of corrupted arterial waveforms using neural network techniques. Comput Biol Med 1992; 22: 173-179

13. Ocasio WC, Rigney DR, Clark KP, Mark RG. bpshapewk4: a computer program that implements a physiological model for analyzing the shape of blood pressure waveforms. Comput Method Prog Biomed 1993; 39: 169-194

14. Kuo TBJ, Chan SHH. Continuous, on-line, real-time spectral analysis of systemic arterial pressure signals. Am J Physiol 1993; 264: H2208-H2213 